\title{
Genetic Stability of a Tick-Borne Encephalitis (TBE) Virus Strain used for the Production of a TBE Vaccine
}

Michael Bröker ${ }^{1 *}$, Markus Eickmann² and Konrad Stadler ${ }^{3}$

${ }^{1}$ Novartis Vaccines and Diagnostics $\mathrm{GmbH}$, Emil-von-Behring-Straße 76, 35041 Marburg, Germany

${ }^{2}$ University of Marburg, Institute of Virology, Hans-Meerwein-Straße 2, 35043 Marburg, Germany

${ }^{3}$ Novartis Vaccines, Via Fiorentina 1, 53100 Siena, Italy, current address: Boehringer Ingelheim Vetmedica GmbH, Binger Strasse 173, 55216 Ingelheim am Rhein, Germany

\begin{abstract}
Tick-borne encephalitis (TBE) is an arthropod-borne virus disease with more than 10,000 cases annually in Europe and Asia. Vaccines have been developed to help protect against TBE. Pre-requisite for consistent production of a viral vaccine is the stable replication of the virus from early development steps to the end of production. We have analysed the nucleotide sequence encoding the surface glycoprotein E of TBE virus strain K23 derived from the virus master seed bank, the working seed bank and a production lot and no sequence variation was found in these three virus samples. We conclude that the genetic constitution during the production process of the analysed TBE vaccine is highly consistent and stable, which is a requirement for defined antigen production process.
\end{abstract}

Keywords: Tick-borne encephalitis; TBE virus; TBE vaccine; Genome analysis; Glycoprotein E

\section{Introduction}

Tick-borne encephalitis (TBE) causes more than 10,000 human cases across Europe and Asia annually. In its natural habitat, the main reservoir of TBE virus (TBEV) are rodents (e.g. mice, moles) and the vector are ticks of the genus Ixodes. Humans are incidental hosts for ticks and may become infected by suckling ticks. The clinical manifestation caused by the TBEV range from inapparent infections and fever with complete recovery of patients to severe clinical pictures and fatal cases $[1,2]$. TBE can be caused by three closely related subtypes of the virus which have a different virulence. The European subtype (TBEV-Eu) is mostly transmitted by hard ticks of the genus I. ricinus, while the Siberian subtype (TBEV-Sib) and the Far Eastern subtype (TBEV-Fe) are mostly transmitted by I. persulcatus [3]. The clinical outcome of TBEV infection in humans depends on the respective TBEV subtype. TBEV-Fe subtype is regarded as the most virulent strain with more than $20 \%$ case fatality rate reported, while the TBEV-Eu subtypes has a case fatality rate of about $1 \%$. The induction of chronic disease has been linked to the TBEV-Sib subtype, which has an intermediate case fatality rate of about $7 \%[1,4]$.

Mature TBE virions are spherical particles with a positive orientated, single-stranded RNA genome of about $11 \mathrm{~kb}$ embedded in a capsid that is surrounded by a lipid envelope in which the glycoproteins $\mathrm{M}(\mathrm{gM})$ and $\mathrm{E}(\mathrm{gE})$ are inserted. The gE plays a pivotal role in the viral life cycle mediating binding of the virus to its cellular receptor (glycosaminoglycans) and fusion of the virus with the cellular membrane. Furthermore, the gE represents the major antigenic determinant of the virus inducing a protective immune response [1] based on neutralizing antibodies broadly cross-protective among the TBEV-Eu, -Sib, and -Fe subtypes $[5,6]$.

Two inactivated whole virus vaccines are internationally available, one is based on the TBEV-Eu subtype strain K23 (originally isolated near Karlsruhe in Southern Germany) and the other vaccine is based on the TBEV-Eu subtype strain Neudörfl (originally isolated in Austria) [7]. Both TBE vaccine manufacturers use primary chick embryonic fibroblast cells (PCEC) for the propagation of the TBE virus and both vaccines are based on purified inactivated virus preparations. The formulations and the history of development of both vaccines as well as their immunogenicity and safety profiles have been reviewed in detail $[7,8]$. These two highly effective modern vaccines are on the market in 28 European countries [9]. In addition to these two widely distributed vaccines, two local manufacturers in Russia and one in China produce TBE vaccines.

Mutations in the gE of a number of flavivirus strains are responsible for changes in host range, cell tropism and altered virulence and attenuation [10-12]. It has been shown that serial passages of viruses in cell culture, especially on cells not derived from the natural host, can result in cell-adapted virus mutants. Some of these mutants show changes in the net charge of their gE proteins, influencing the affinity for glycosaminoglycans which are putative viral receptors on the cell surface [10]. Those changes in the molecular structure of the $\mathrm{gE}$ molecule can result in an altered virulence in vivo and may be caused by a single amino acid exchange [11]. Even more important for vaccine manufacturers, due to mutations in the $\mathrm{gE}$, otherwise protective epitopes might get modified or lost and thus, decreasing the protective efficacy of a vaccine. Here, we describe investigations to analyse the stability of the genetic information encoding the gE of TBEV strain K23 during the vaccine manufaturing process, starting from the viral master seed lot to the final product.

\section{Material and Methods}

The TBE virus strain K23 had been isolated by Prof. R. Ackermann, University of Cologne, from ticks collected in Southern Germany near Karlsruhe in 1975 [13]. The strain K23 was received as the third passage of the virus in the brains of suckling mice and was further propagated

*Corresponding author: Michael Bröker, Novartis Vaccines and Diagnostics GmbH, Emil-von-Behring-Str. 76, 35041 Marburg, Germany, Tel: +49 6421392912 Fax: +49 6421 394667; E-mail: Michael.Broeker@Novartis.com

Received January 13, 2011; Accepted February 12, 2011; Published February 20, 2011

Citation: Bröker M, Eickmann M, Stadler K (2011) Genetic Stability of a TickBorne Encephalitis (TBE) Virus Strain used for the Production of a TBE Vaccine. J Vaccines Vaccin 2:114. doi:10.4172/2157-7560.1000114

Copyright: @ 2011 Bröker M, et al. This is an open-access article distributed under the terms of the Creative Commons Attribution License, which permits unrestricted use, distribution, and reproduction in any medium, provided the original author and source are credited. 
in primary chick embryo fibroblasts (PCEC). The $20^{\text {th }}$ passage on PCEC was carried out in a bigger quantity and was used as master seed. This master seed lot was produced in January 1987 and was stored at $-80^{\circ} \mathrm{C}$ in suitable containers. This lot was intensively tested for absence of adventitious agents, purity and identity in accordance with WHO and national requirements [14]. A working seed bank was produced in September 1995 diverged from the Master Seed Bank produced in 1987 by propagating on PCEC and stored in suitable aliquots at $-80^{\circ} \mathrm{C}$. The production process of the TBE vaccine has been described in detail by Klockmann et al. [14].

TBEV material used is this study was i) a master seed bank prepared in January 1987 and stored for 17 years, ii) the working seed bank prepared in September 1995 and stored for 9 years, and iii) material from the end of a production run in November 2004.

For the 3 isolates sequenced in this study, viral RNA was extracted from the master and working seed lot and from a vaccine batch using the QIAamp Viral RNA Mini Kit (Qiagen) and was reverse transcribed with the SuperScript II Reverse Transcriptase kit (Invitrogen, Carlsbad, CA - USA). PCR amplifications were performed by using specific primers (primer sequences available on request) and the products were purified by use of a QIAquick PCR purification kit (Qiagen), according to the manufacturer's directions. The Taq polymerase and other reagents used for PCR were obtained from Qiagen. The sequences were obtained by direct sequencing of the PCR products, which was performed by the DNA-sequencing facility at Novartis Vaccines, Siena, Italy. Sequence data were assembled and edited with Vector NTI software (Invitrogen) and compared with representative sequences of viruses available in GenBank (http://www.ncbi.nlm.nih.gov/genbank). The nucleotide sequences obtained in this study are deposited in gene bank of the European Molecular Biology Laboratories Sequence database URL http://www.ebi.ac.uk/embl with the accession number AM600965 and at http://www.ncbi.nlm.nih.gov/nuccore/AM600965.

\section{Results and Discussion}

Two internationally available TBE vaccines have been developed either based on TBEV-Eu strain K23 or strain Neudörfl and which confer protection not only against the TBEV-Eu subtype, but also against strains representing the Siberian and Far Eastern subtypes. Neutralizing antibodies against the E protein is a correlate of protection against virus infection [1]. Both virus strains have been isolated from ticks and were adapted to growth on chick embryo fibroblasts.

Sequence analysis of the gene encoding the gE protein of the master and working seed lot virus and of a vaccine batch revealed no differences in the $\mathrm{gE}$ protein of TBE virus K23. Comparison of the E protein from K23 TBE virus with another subtype of TBEV-Eu, strain Neudörfl published by Ecker et al. [3], disclosed four amino acid exchanges at positions 52: lysine $(\mathrm{K})$ to asparagine $(\mathrm{N}), 84$ : threonine $(\mathrm{T})$ to alanine (A), 137: arginine (R) to lysine (K). At position 167, valine (V) was determined for both strains K23 and Neudörfl, while in the original publication by Ecker et al. [3], the authors indicated an isoleucine (I) at this position (Figure 1).

Sequencing of vaccine strains is of interest from two points. First, it is well-known that changes in neurovirulence and pathogenicity of the virus can be due to even a single amino acid exchanges and changes in an important epitope may reduce or destroy the capacity to induce neutralizing antibodies to the homologous and/or heterologous strains. Otherwise, mutations may also result in an increase in virulence. In this context, it is notable, that a strain has recently been isolated in a new TBE focus in Bavaria, Germany, where three of nine clinical human

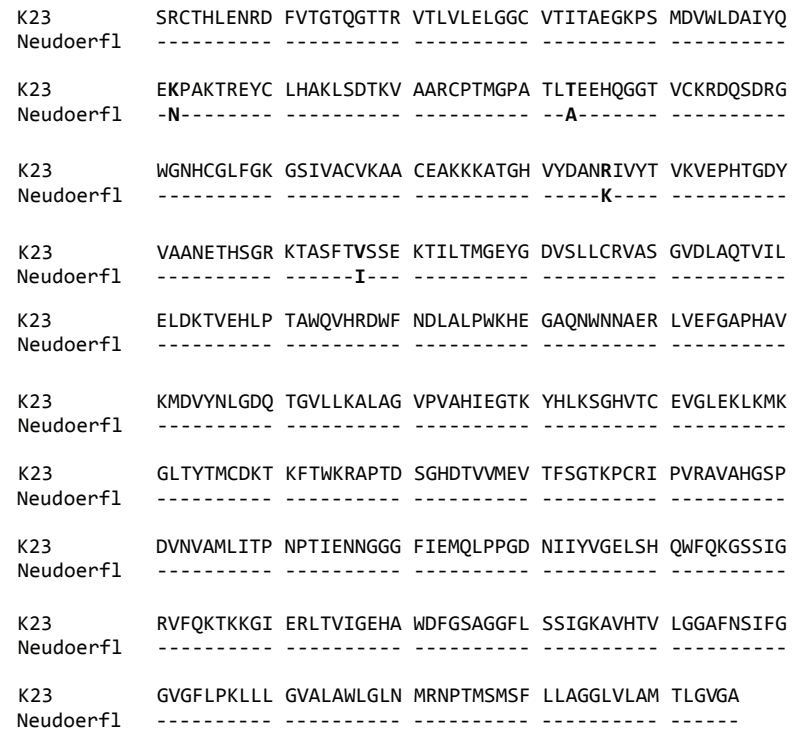

Neudoerfl: NCBI NC_001672

K23: Genbank AM600965.1

Figure 1: Amino acid sequence of K23 strain isolated from master seed bank, working seed bank and from the end of the production process compared to prototype sequence of strain Neudoerfl.

cases were fatal indicating an unusually high mortality rate possibly due to a highly virulent TBEV strain. Sequencing of the virus isolate from this focus revealed two unique amino acid substitutions in the envelope protein, known to play a role in the pathogenicity of TBE virus and the membrane fusion with host cells [15].

Genetic instability could cause an altered antigen make-up with reduced efficacy of the vaccine produced by a mutated strain. Second, upon development of master and working seed lots for the production of a vaccine, clinical studies are carried out to evaluate safety and immunogenicity. These seed lots are then used for many years upon licensure of the vaccine. Quality control tests include invitro and in-vivo analyses, e.g. evaluation of immunogenicity and efficacy in challenge experiments, but analyses on the molecular basis are commonly not carried out, because these are not requested by the authorities. Therefore, we were interested to analyse the genomic sequence, especially the coding gene for the glycoprotein E gene that comprises neutralizing capacity and is of upmost importance for the efficacy of the vaccine from a master seed lot and a working seed lot with a history of 17 and 9 years respectively and of a virus sample harvested at the end of the production phase. Comparison of the compiled sequences revealed no nucleotide changes during the long production history of the K23 based TBE vaccine and therefore this indicates a consistent and stable virus handling procedure in the virus storage and vaccine production process.

\section{Conflict of Interest}

M.B. is a full time employee of Novartis Vaccines. KS was full time employee of Chiron Vaccines (the ancestor company of Novartis Vaccines) when these studies were carried out. ME declares no conflict of interest.

\section{References}

1. Dumpis U, Crook D, Oksi J (1999) Tick-borne encephalitis. J Clin Infect Dis 28: $882-890$.

2. Bogovic P, Lotric-Furlan S, Strle F (2010) What tick-borne encephalitis may look like: clinical signs and symptoms. Travel Med Infect Dis 8: 246-250. 
Citation: Bröker M, Eickmann M, Stadler K (2011) Genetic Stability of a Tick-Borne Encephalitis (TBE) Virus Strain used for the Production of a TBE Vaccine. J Vaccines Vaccin 2:114. doi:10.4172/2157-7560.1000114

3. Ecker M, Allison SL, Meixner T, Heinz FX (1999) Sequence analysis and genetic classification of tick-borne encephalitis viruses from Europe and Asia. J Gen Virol 80: 179-185.

4. Dörrbecker B, Dobler G, Spiegel M, Hufert F (2010) Tick-borne encephalitis virus and the immune response of the mammalian host. Travel Med Infect Dis 8: 213-222.

5. Leonova G, Ternovoi VA, Pavlenko EV, Maistrovskaya OS, Protopopova EV, et al. (2007) Evaluation of vaccine Encepur ${ }^{\circledR}$ Adult for induction of human neutralizing antibodies against recent Far Eastern subtype strains of tick-borne encephalitis virus. Vaccine 25: 895-901.

6. Hayasaka D, Goto A, Yoshii K, Mizutani T, Kariwa H, et al. (2001) Evaluation of European tick-borne encephalitis virus vaccine against recent Siberian and far-eastern subtype strains. Vaccine 19: 4774-4779.

7. Zent O, Bröker M (2005) Tick-borne encephalitis vaccines: past and present. Expert Rev Vaccines 4: 747-755.

8. Barret NP, Schober-Bendixen S, Ehrlich HJ (2003) History of TBE vaccines. Vaccine 21: 41-49.

9. Wiedermann $U$ (2010) Tick-borne encephalitis TBE - Vaccination in nonendemic countries. Travel Med Infect Dis 8: 251-246.
10. Lee E, Wright PJ, Davidson A, Lobigs M (2006) Virulence attenuation of Dengue virus due to augmented glycosaminoglycan-binding affinity and restriction in extraneural dissemination. J Gen Virol 87: 2791-2801.

11. Goto A, Hayasaka D, Yoshii K, Mizutani T, Kariwa H, et al. (2003) A BHK-21 cell-culture-adapted tick-borne encephalitis virus mutant is attenuated for neuroinvasiveness. Vaccine 21: 4043-4051.

12. Khasnatinov MA, Ustanikova K, Frolova TV, Pogodina VV, Bochkova NG, et al. (2009) Non-haemaglutionating flaviviruses. Molecular mechanisms for the emergence of new strains via adaption to European ticks. PLoS ONE 4: e7295.

13. Rehse-Küpper B, Danielova V, Klenk W, Abar B, Ackermann R (1978) The isolation of Central European Encephalitis (tick-borne encephalitis) virus from Ixodes ricinus (L.) ticks in southern Germany. Zentralbl Bakteriol Orig A 242 148-155.

14. Klockmann U, Bock HL, Franke B, Hein B, Reiner G, et al. (1989) Preclinical investigations of the safety, immunogenicity and efficacy of a purified inactivated tick-borne encephalitis vaccine. J Biol Standard 17: 331-342.

15. Kupča AM, Essbauer S, Zoeller G, de Mendonça PG, Brey R, et al. (2010) Isolation and molecular characterization of a tick-borne encephalitis virus strain from a new tick-borne encephalitis focus with severe cases in Bavaria, Germany. Ticks Tick-borne Dis 1: 44-51. 